\title{
A Rare Coincidence of Sitosterolemia and Familial Mediterranean Fever Identified by Whole Exome Sequencing
}

\author{
Hayato Tada1, Masa-aki Kawashiri ${ }^{1}$, Hirofumi Okada ${ }^{1}$, Saori Endo², Yuka Toyoshima², Tetsuo Konno ${ }^{1}$, \\ Atsushi Nohara ${ }^{1}$, Akihiro Inazu ${ }^{3}$, Akira Takao ${ }^{2}$, Hiroshi Mabuchi ${ }^{1}$, Masakazu Yamagishi ${ }^{1}$ and Kenshi Hayashi ${ }^{1}$ \\ ${ }^{1}$ Division of Cardiovascular Medicine, Kanazawa University Graduate School of Medicine, Kanazawa, Japan \\ ${ }^{2}$ Department of Pediatrics, Gifu Municipal Hospital, Gifu, Japan \\ ${ }^{3}$ Department of Clinical Laboratory Science, Faculty of Health Sciences, Institute of Medical, Pharmaceutical and Health Sciences, \\ Kanazawa University, Kanazawa, Japan
}

\begin{abstract}
Whole exome sequencing (WES) technologies have accelerated genetic studies of Mendelian disorders, yielding approximately 30\% diagnostic success. We encountered a 13-year-old Japanese female initially diagnosed with familial hypercholesterolemia on the basis of clinical manifestations of severe hypercholesterolemia (initial LDL cholesterol $=609 \mathrm{mg} / \mathrm{dl}$ at the age of one) and systemic intertriginous xanthomas with histories of recurrent self-limiting episodes of fever and arthritis. Both her phenotypes seemed to co-segregate in a recessive manner. We performed WES on this patient, who was considered a proband. Among 206,430 variants found in this individual, we found 18,220 nonsense, missense, or splice site variants, of which 3,087 were rare (minor allele frequency $\leq 0.01$ or not reported) in 1000 Genome (Asian population). Filtering by assuming a recessive pattern of inheritance with the use of an in silico annotation prediction tool, we successfully narrowed down the candidates to the compound heterozygous mutations in the ABCG5 gene (c.1256G > A or p.Arg419His/ c.1763-1G > A [splice acceptor site]) and to the double-compound heterozygous mutations in the $M E F V$ gene (c.329T $>$ C/C or p.Leu110Pro/c.442G $>C / C$ or p.Glu148Val). The patient was genetically diagnosed with sitosterolemia and familial Mediterranean fever using WES for the first time. Such a comprehensive approach is useful for identifying causative mutations for multiple unrelated inheritable diseases.
\end{abstract}

J Atheroscler Thromb, 2016; 23: 884-890.

Key words: Sitosterolemia, Familial Mediterranean fever, $A B C G 5, A B C G 8$, Whole exome sequencing

\section{Introduction}

Sitosterolemia (OMIM \#210250) caused by mutations in either of two genes ATP-binding cassette (ABC) sub-family $\mathrm{G}$ members 5 and 8 ( $A B C G 5$ and $A B C G 8)$ is an extremely rare autosomal recessive disorder of sterol metabolism characterized by increased

Address for correspondence: Hayato Tada, Division of Cardiovascular Medicine, Kanazawa University Graduate School of Medicine, 13-1 Takara-machi, Kanazawa, 9208641, Japan.

Email: ht240z@sa3.so-net.ne.jp

Received: January 13, 2016

Accepted for publication: March 22, 2016 absorption and decreased biliary excretion of plant sterols and cholesterol, resulting in prominently elevated serum levels of plant sterols such as sitosterol and campesterol $^{1,2)}$. Subjects suffering from sitosterolemia primarily present tendinous and tuberous xanthomas and premature coronary atherosclerosis resembling familial hypercholesterolemia $(\mathrm{FH})^{3)}$. Familial Mediterranean fever (FMF, OMIM \#249100), which is caused by mutations in the pyrin $(M E F V)$ gene, is an autosomal recessive disorder characterized by recurrent attacks of fever and inflammation in the peritoneum, synovium, or pleura and is accompanied by pain ${ }^{4,5)}$.

We encountered a Japanese female initially diagnosed with $\mathrm{FH}$ on the basis of clinical manifestations 
of severe hypercholesterolemia and systemic intertriginous xanthomas with histories of recurrent self-limiting episodes of fever and arthritis. Both phenotypes seemed to co-segregate in a recessive manner. We therefore tried to establish a comprehensive molecular diagnosis for this rare condition using whole exome sequencing (WES).

\section{Case Report}

\section{Study Subjects}

A 13-year-old Japanese female was referred to our lipid clinic due to her extremely high levels of LDL cholesterol, without any apparent secondary causes. Her LDL cholesterol at the age of one was 609 $\mathrm{mg} / \mathrm{dl}$ when she was under breastfeeding. Both her parents showed no evidence of consanguineous marriage, and her younger sister was also included in this study.

\section{Ethical Considerations}

This study was approved by the Ethics Committee of Kanazawa University and conducted in accordance with the Declaration of Helsinki (2008) of the World Medical Association. All procedures followed were in accordance with the ethical standards of the responsible committee on human experimentation (institutional and national) and with the Helsinki Declaration of 1975, as revised in 2008. Informed consents were obtained from all subjects for being included in the study.

\section{Biochemical Analysis}

Blood samples were drawn for assays after overnight fasting. Serum levels of total cholesterol, triglycerides, and HDL cholesterol were determined enzymatically ${ }^{6}$. Serum levels of sterols, including sitosterol, lathosterol, and campesterol, were determined using gas-liquid chromatography-mass spectrometry as previously described ${ }^{2}$.

\section{Exome Sequencing and Bioinformatics}

Exome capture and sequencing were performed as previously described ${ }^{7}$. We applied three independent filters after standard variant quality controls in order to successfully discover a causal variant among the proband as previously described ${ }^{7)}$. We filtered out the variants as 1) benign variants predicted by SnpEff, 2) minor allele frequency (MAF) $>1 \%$ in an Asian population, and 3) unmatched segregation under the assumption of a recessive form of inheritance.

For the sample, paired-end reads were aligned using the Burrows-Wheeler Aligner on the human ref- erence genome build hg19 using quality score calibration, soft clipping, and adapter trimming. Following the exclusion of PCR duplicate reads using Picard, insertion-deletions and single-nucleotide polymorphisms (SNPs) were identified using GATK $^{8,9)}$. Variants (SNPs/indels) were filtered out on the basis of the Phred-scaled genotype quality score. Re-alignment was performed, and a calling algorithm was used to merge the output of the GATK UnifiedGenotyper. All samples were annotated using SnpEff version 3.6 to classify variants (e.g., missense, nonsense, splice site, synonymous, intronic, or stop gain/loss) ${ }^{10)}$.

Functional filtering, missense, nonsense, and splice site variants were considered as candidate variants. The frequency filter was uses to quantify the allele frequency estimates from the Asian cohort database in the 1000 Genomes Project, and a MAF > $1 \%$ was used as the cut-off. Segregation pattern matching was defined as that the situation where the affected exhibited homozygous or compound heterozygous of alternative alleles in a particular gene. We also filtered out variants with the use of an in silico annotation prediction tool as described previously ${ }^{7}$. Following those evaluations, the putative variants identified by bioinformatics were confirmed using Sanger sequencing methods as previously described ${ }^{11)}$.

\section{Characteristics of Study Subjects}

The clinical characteristics of the study subjects are shown in Table 1. The proband (II.1) initially visited a hospital due to her intertriginous xanthomas in her legs at one year of age. At that time she was diagnosed with $\mathrm{FH}$ based on her having extremely high levels of LDL cholesterol as well as on physical findings. She was thought to have heterozygous $\mathrm{FH}$, because her LDL cholesterol dramatically reduced to around $140 \mathrm{mg} / \mathrm{dl}$ with a small dose of a standard statin $(5 \mathrm{mg}$ pravastatin or $5 \mathrm{mg}$ simvastatin) and because she was weaned from breastfeeding (Fig. 1). At the age of 13 she was referred to Kanazawa University Hospital to undergo a genetic diagnosis, because it was quite unusual to observe such a great reduction in LDL cholesterol in a patient with $\mathrm{FH}$ using such therapies.

At the time of genetic diagnosis, the patient had already exhibited Achilles' tendon thickness (Fig. 2), thickness in her carotid artery (Fig. 3), and moderate aortic regurgitation associated with aortic valve calcification (Fig. 4). Her father was taking statin $(2.5 \mathrm{mg}$ rosuvastatin) without any physical xanthomas or any family history of premature coronary artery disease. Her mother exhibited normolipidemia. Based on the recessive pattern of inheritance as well as the great 
Table 1. Characteristics of the study subjects

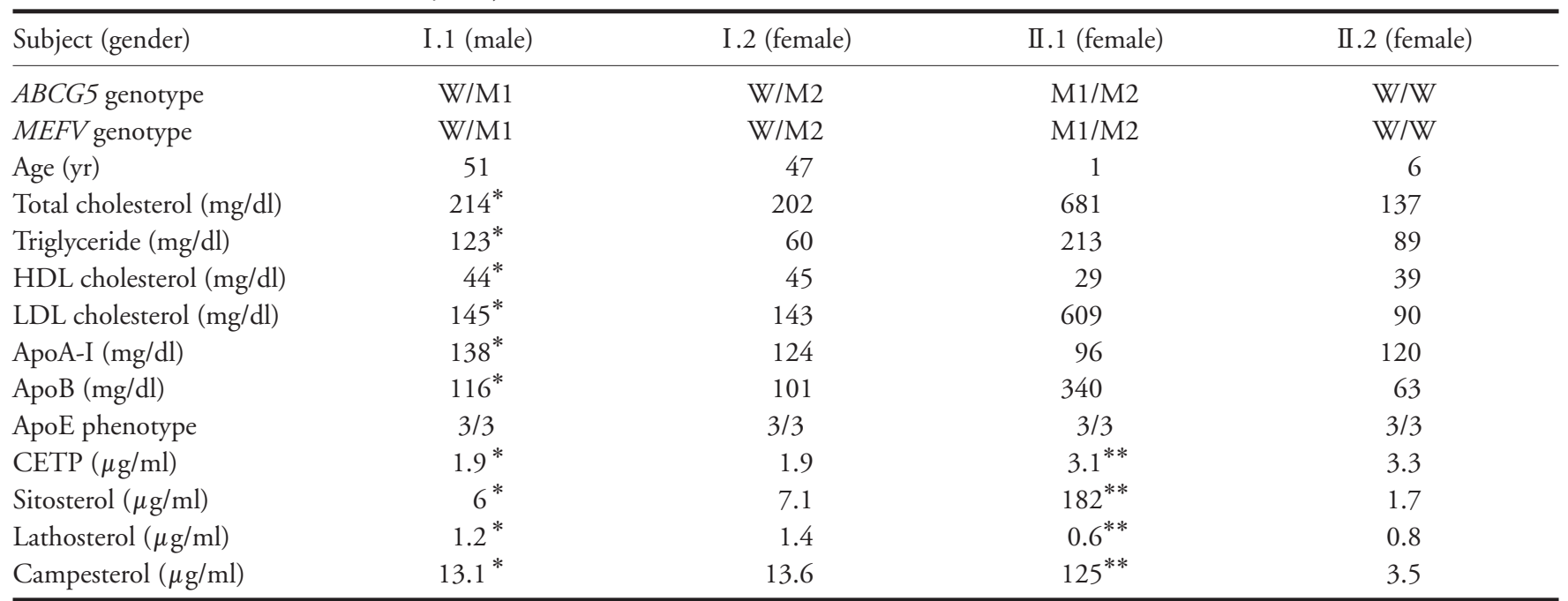

ABCG5: ATP-binding cassette sub-family G member 5, MEFV: Mediterranean fever, CETP: cholesteryl ester transfer protein

$A B C G 5$ genotype: $\mathrm{W}=$ wild type, $\mathrm{M} 1=\mathrm{c} .1256 \mathrm{G}>\mathrm{A}, \mathrm{M} 2=\mathrm{c} .1763-1 \mathrm{G}>\mathrm{A}, \mathrm{MEFV}$ genotype: $\mathrm{W}=$ wild type, $\mathrm{M} 1=\mathrm{c} .329 \mathrm{~T}>\mathrm{C} / \mathrm{C}, \mathrm{M} 2=\mathrm{c} .442 \mathrm{G}>$ $\mathrm{C} / \mathrm{C}$

*Values measured when rosuvastatin was $2.5 \mathrm{mg}$.

** CETP, sitosterol, lathosterol, and campesterol were measured at the age of 13 (when rosuvastatin was $5 \mathrm{mg}$ ).

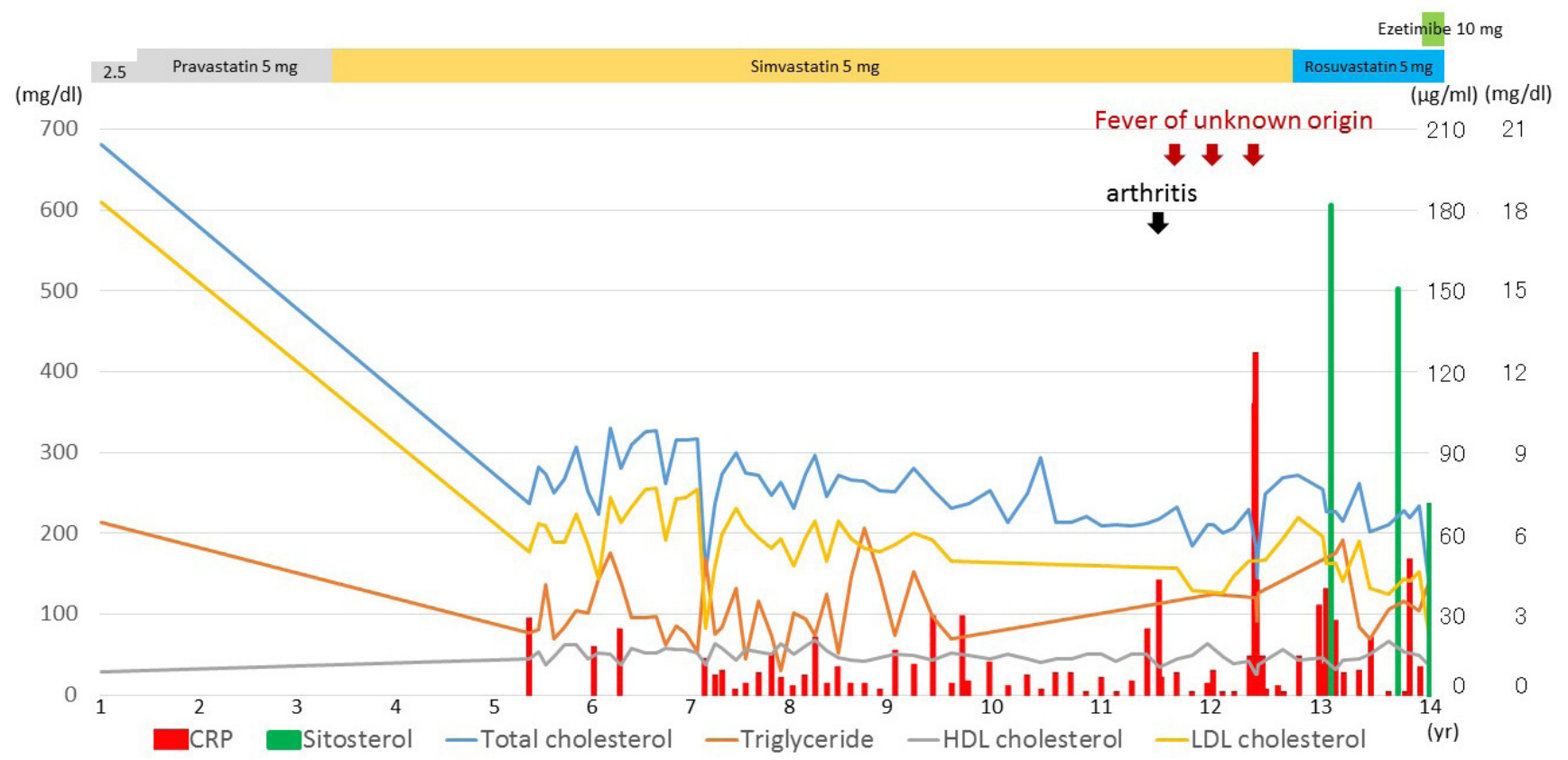

Fig. 1. Clinical course of the proband

$\mathrm{X}$-axis indicates age. Line graphs on the Y-axis (left side) indicate total cholesterol, triglycerides, HDL cholesterol, and LDL cholesterol in mg/ dl. Bar graphs on the Y-axis (right side) indicate CRP in $\mathrm{mg} / \mathrm{dl}$, and sitosterol in $\mu \mathrm{g} / \mathrm{ml}$.

reduction in LDL cholesterol, we suspected her condition to be sitosterolemia, and thus measured her serum sitosterol. Her initial serumal levels of sitosterol at the age of 13 was as high as $182 \mu \mathrm{g} / \mathrm{ml}$, confirming her clinical diagnosis as sitosterolemia. Her blood counts were within normal range. Her sitosterol levels could be reduced to $70 \mu \mathrm{g} / \mathrm{ml}$ using $10 \mathrm{mg}$ ezetimibe in accordance with a previous report ${ }^{2}$. We also 


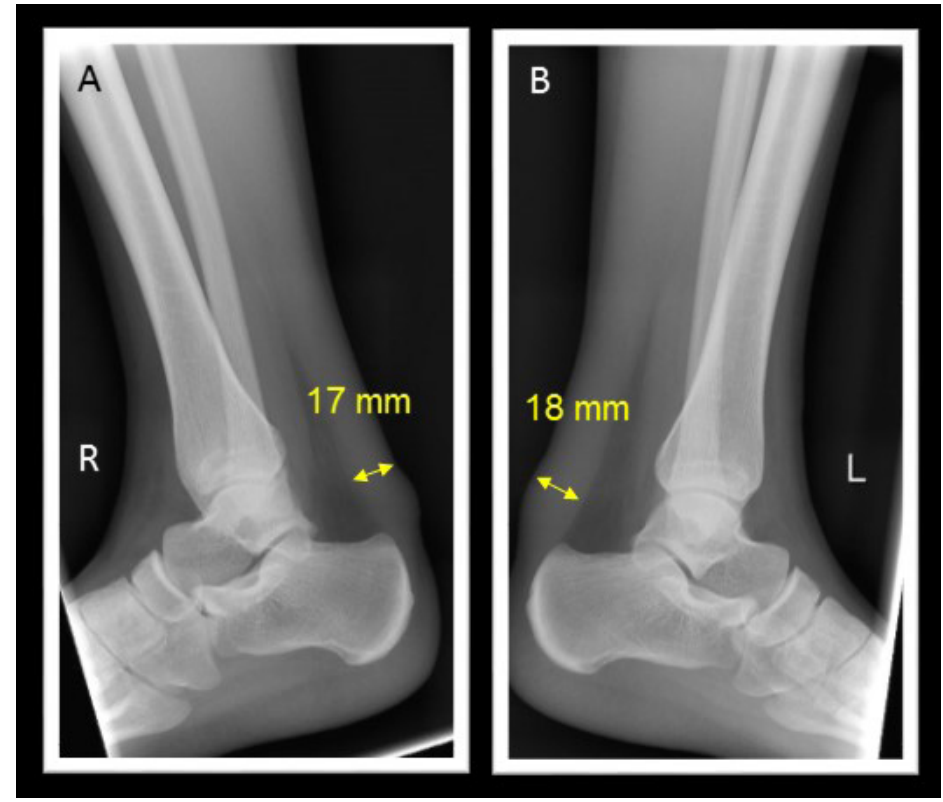

Fig. 2. X-ray for Achilles' tendon

A. Right. B. Left

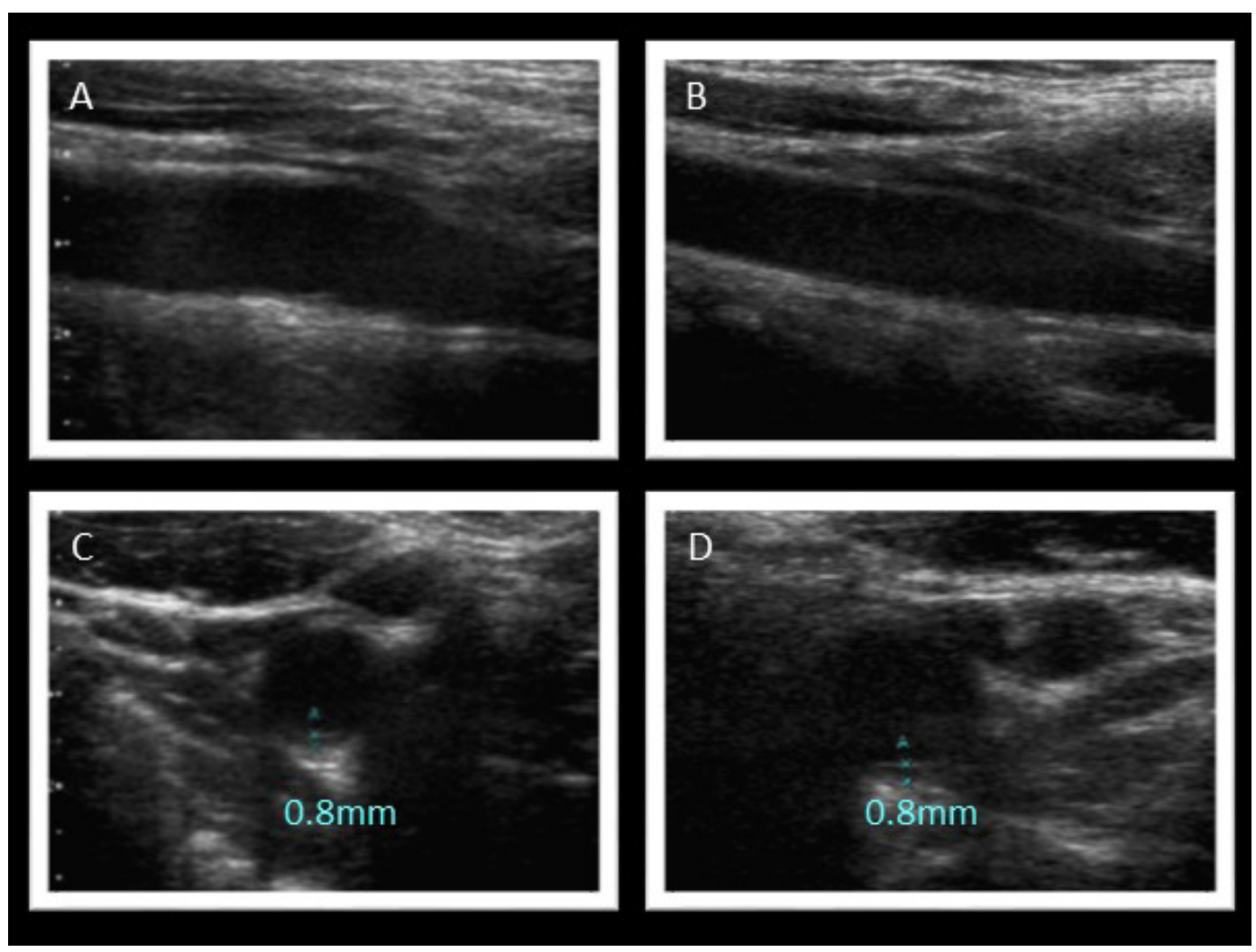

Fig. 3. Ultrasound images of carotid arteries

A. Long-axis view (right). B. Long-axis view (left). C. Short-axis view (right). D. Short-axis view (left). Bilateral carotid intima-media thickness was $0.8 \mathrm{~mm}$. 


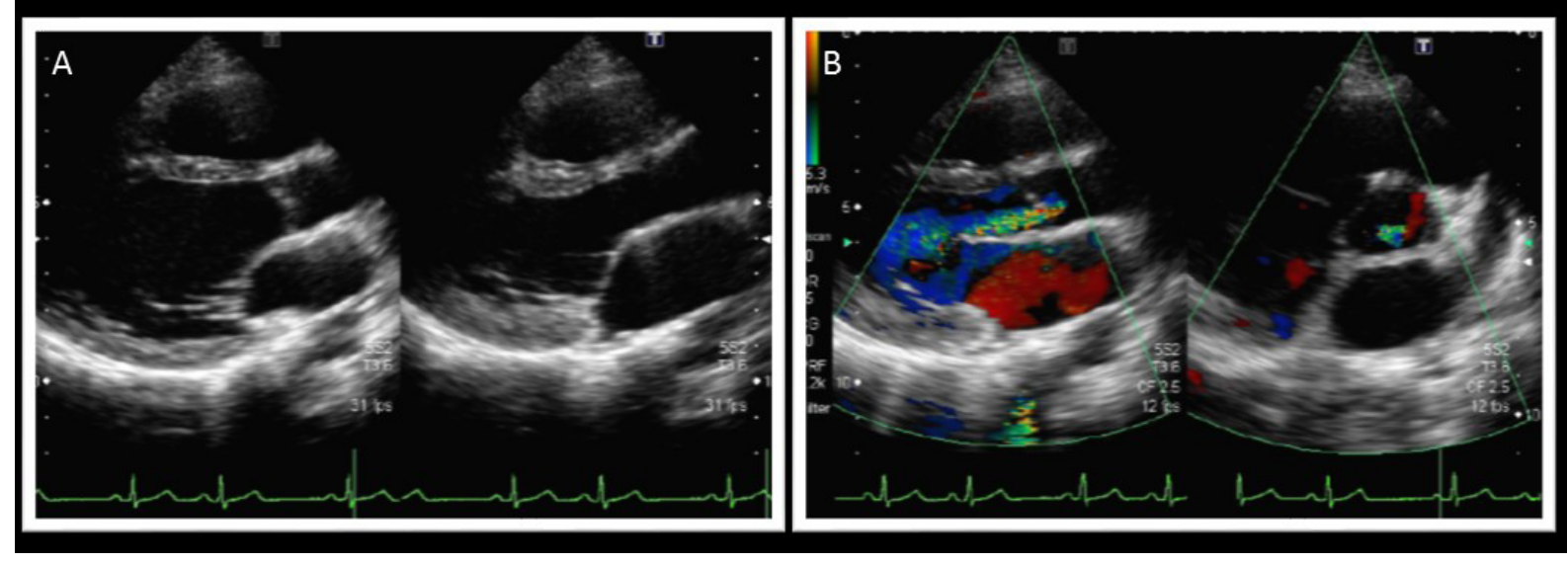

Fig. 4. Echocardiography

A. Long-axis view (plain). B. Long- and short-axis view (in color). Echocardiography revealed moderate aortic regurgitation associated with aortic valve calcification.

observed great reduction in her LDL cholesterol from $156 \mathrm{mg} / \mathrm{dl}$ to $76 \mathrm{mg} / \mathrm{dl}$ using $10 \mathrm{mg}$ of ezetimibe. In addition, she had experienced several occurrences of high grade fever and arthritis associated with elevated C-reactive protein (Fig. 1).

\section{Exome Sequencing and Bioinformatics Analysis}

The proband underwent whole exome sequencing, and subsequent bioinformatics filtering. The mean depth was $99.9 \times$ per base across the whole exome. The percentage of on-target reads was $84.6 \%$. Also, the coverage rate of target coding lesions $(10 \times)$ was $99.0 \%$.

The number of aligned variants in the proband that passed through standard quality control was 206,430. Of those, 18,220 were missense, nonsense, splice site, or frameshift variants. After removing "common" variants with MAF > $1 \%$ using the Asian cohort in the 1000 genome project ${ }^{12)}$, 3,087 variants were detected. Subsequently, filtering against the segregation pattern while assuming the recessive form of inheritance with the use of in silico annotation prediction tool reduced the candidate variants to compound heterozygous mutations in the $A B C G 5$ gene (c.1256G $>\mathrm{A}$ or p.Arg419His/c.1763-1G >A [splice acceptor site]) on chromosome 2 and double compound heterozygous mutations in the $M E F V$ gene (c.329 T $>\mathrm{C} /$ C or p.Leu110Pro/c.442G $>$ C/C or p.Glu148Val) on chromosome 16. The former mutation in the $A B C G 5$ gene is one of the most common mutations among Asians with sitosterolemia ${ }^{13)}$, while the latter mutation in the $A B C G 5$ gene is considered novel. Meanwhile, both mutations in the $M E F V$ gene identified in the proband are two of the most common mutations among Japanese patients with $\mathrm{FMF}^{14)}$. The status of mutations in her family members (Fig. 5A) was determined by subsequent Sanger sequencing (Fig. 5B-5E). Finally, we diagnosed her as having sitosterolemia and FMF; this is considered to be a rare condition of sitosterolemia. We consider this to be the first time such a condition has been diagnosed using an exome-wide approach.

\section{Discussion}

Using a WES approach, we identified the first case of a rare coincidence of sitosterolemia and FMF exhibiting compound heterozygous mutations in the $A B C G 5$ gene and double compound heterozygous mutations in the $M E F V$ gene. To the best of our knowledge, this is the first report of this coincidence. These findings allow us to draw several conclusions. First, the WES approach is useful to identify causative mutations in a family with a recessive form of inheritance, even when DNA of only one affected individual is available. Second, we can determine true causative mutations not from target re-sequencing, but from an exome-wide approach. Third, such a comprehensive approach is useful in identifying causative mutations for multiple unrelated inherited diseases.

In terms of mode of inheritance, her father exhibited mildly elevated levels of LDL cholesterol under statin therapy without any evidence of extreme hypercholesterolemia or cutaneous xanthomas. There was no deleterious mutation in the coding lesion of the LDL receptor or in the PCSK9 gene in her father; this led us to the notion of recessive inheritance.

Thus far, only 100 or so patients have been diag- 
A

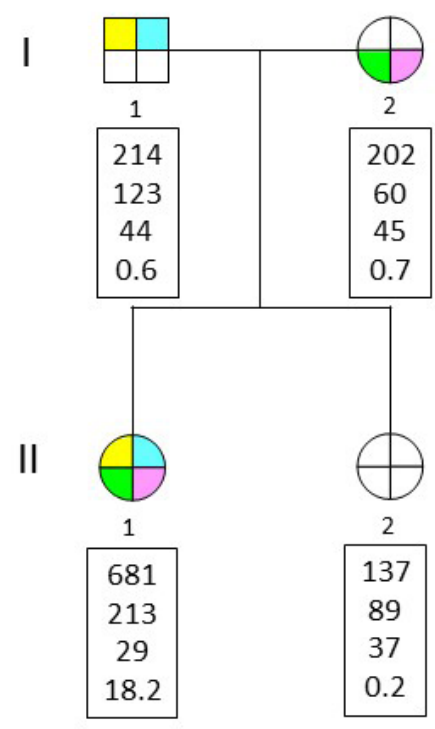

\begin{tabular}{|l|}
\hline Total cholesterol $(\mathrm{mg} / \mathrm{dl})$ \\
Triglyceride $(\mathrm{mg} / \mathrm{dl})$ \\
HDL cholesterol $(\mathrm{mg} / \mathrm{dl})$ \\
Sitosterol $(\mathrm{mg} / \mathrm{dl})$
\end{tabular}

\begin{tabular}{|lcc|}
\hline$\boxplus$ & $\oplus$ & c.1256G>A (ABCG5) carrier \\
$\boxplus$ & $\oplus$ & c.1763-1G>A (ABCG5) carrier \\
$\boxplus$ & $\oplus$ & c.329T>C/C (MEFV) carrier \\
$\boxplus$ & $\oplus$ & c.442G>C/C (MEFV) carrier \\
$\bigcirc$ & female $\quad \square$ male \\
\hline
\end{tabular}
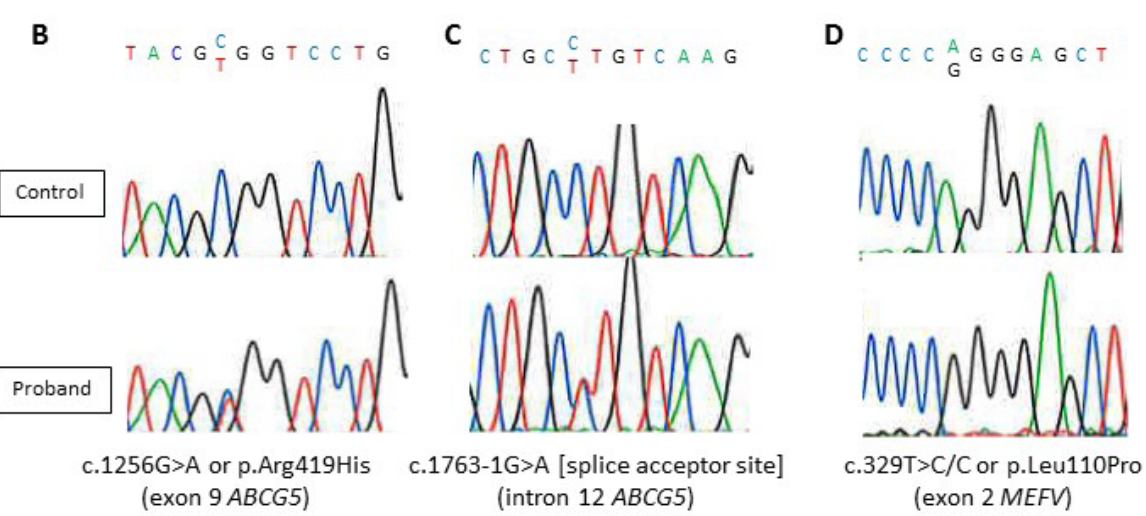

E $C C C G_{G}^{C} A G G C C G G G$

Fig. 5. Family tree and mutation validation

A. Yellow indicates the carrier of c. $1256 \mathrm{G}>\mathrm{A}$ or p.Arg419His mutation in the $A B C G 5$ gene. Green indicates the carrier of the c.1763-1G $>$ A mutation in the $A B C G 5$ gene. Blue indicates the carrier of the c.329 $\mathrm{T}>\mathrm{C} / \mathrm{C}$ or p.Leu110Pro mutation in the $M E F V$ gene. Pink indicates the carrier of the c. $442 \mathrm{G}>\mathrm{C} / \mathrm{C}$ or p.Glu148Val mutation in the $M E F V$ gene.

B-E. Mutations were validated by Sanger sequencing.

nosed with sitosterolemia ${ }^{15}$. The clinical features of sitosterolemia includes xanthomas, premature atherosclerosis, and increased serum levels of plant sterols ${ }^{1,2)}$. In addition, they may exhibit extreme hypercholesterolemia induced by breastfeeding in accordance with our previous findings in infantile cases with sitosterolemia $^{2)}$. Separately, FMF, which is characterized by recurrent, self-limiting episodes of fever, affects more than 100,000 patients, primarily in the Mediterranean Basin ${ }^{16)}$. The proband in this study was diagnosed with $\mathrm{FH}$ and was treated with statins; however, ezetimibe could be a much more reasonable therapy for her based on her recent diagnosis with sitosterol- emia $^{17-19)}$.

Careful monitoring and additional therapeutic approaches are needed to reduce her serum levels of sitosterol because of the unfavorable combination of hypersitosterolemia and systemic inflammatory disease, both of which have been shown to be associated with an increased risk of cardiovascular disease.

We report a case exhibiting a rare coincidence of sitosterolemia and FMF identified using WES. Such a comprehensive approach is useful in identifying causative mutations for multiple unrelated inherited diseases. 


\section{Source of Finding}

A scientific research grant from the Ministry of Education, Science, and Culture of Japan (No. 26893094).

\section{Acknowledgments}

None.

\section{Conflict of Interest Statement}

None.

\section{References}

1) Shulman RS, Bhattacharyya AK, Connor WE, Fredrickson DS: Beta-sitosterolemia and xanthomatosis. N Engl J Med, 1976; 294: 482-483

2) Tada H, Kawashiri MA, Takata M, Matsunami K, Imamura A, Matsuyama M, Sawada $\mathrm{H}$, Nunoi $\mathrm{H}$, Konno T, Hayashi K, Nohara A, Inazu A, Kobayashi J, Mabuchi H, Yamagishi M: Infantile cases of sitosterolaemia with novel mutations in the ABCG5 gene: Extreme hypercholesterolaemia is exacerbated by breastfeeding. JIMD Rep, 2015; 21: $115-122$

3) Salen G, Patel S, Batta AK: Sitosterolemia. Cardiovasc Drug Rev, 2002; 20: 255-270

4) Sohar E, Gafni J, Pras M, Heller H: Familial Mediterranean fever. A survey of 470 cases and review of the literature. Am J Med, 1967; 43: 227-253

5) French FMF Consortium: A candidate gene for familial Mediterranean fever. Nat Genet, 1997; 17: 25-31

6) Tada H, Kawashiri MA, Nohara A, Inazu A, Mabuchi H, Yamagishi M, Hayashi K: Lipoprotein metabolism in familial hypercholesterolemia: Serial assessment using a one-step ultracentrifugation method. Practical Lab Med, 2015; 1: 22-27

7) Tada H, Kawashiri MA, Nohara A, Saito R, Tanaka Y, Nomura A, Konno T, Sakata K, Fujino N, Takamura T, Inazu A, Mabuchi H, Yamagishi M, Hayashi K: Whole exome sequencing combined with integrated variant annotation prediction identifies asymptomatic Tangier disease with compound heterozygous mutations in $\mathrm{ABCA} 1$ gene. Atherosclerosis, 2015; 240: 324-329

8) McKenna A, Hanna M, Banks E, Sivachenko A, Cibulskis K, Kernytsky A, Garimella K, Altshuler D, Gabriel S, Daly M, DePristo MA: The Genome Analysis Toolkit: a MapReduce framework for analyzing next-generation DNA sequencing data. Genome Res, 2010; 20: $1297-$ 1303

9) DePristo MA, Banks E, Poplin R, Garimella KV, Maguire JR, Hartl C, Philippakis AA, del Angel G, Rivas MA, Hanna M, McKenna A, Fennell TJ, Kernytsky AM, Sivachenko AY, Cibulskis K, Gabriel SB, Altshuler D, Daly
MJ: A framework for variation discovery and genotyping using next-generation DNA sequencing data. Nat Genet, 2011; 43: 491-498

10) Cingolani P, Platts A, Wang le L, Coon M, Nguyen T, Wang L, Land SJ, Lu X, Ruden DM: A program for annotating and predicting the effects of single nucleotide polymorphisms, SnpEff: SNPs in the genome of Drosophila melanogaster strain w1118; iso-2; iso-3. Fly (Austin) 6 (2012) 80-92

11) Tada H, Kawashiri MA, Ikewaki K, Terao $Y$, Noguchi $T$, Nakanishi C, Tsuchida M, Takata M, Miwa K, Konno T, Hayashi K, Nohara A, Inazu A, Kobayashi J, Mabuchi H, Yamagishi M: Altered metabolism of low-density lipoprotein and very-low-density lipoprotein remnant in autosomal recessive hypercholesterolemia: results from stable isotope kinetic study in vivo. Circ Cardiovasc Genet, 2012; 5: 35-41

12) 1000 Genomes Project Consortium, Abecasis GR, Altshuler D, Auton A, Brooks LD, Durbin RM, Gibbs RA, Hurles ME, McVean GA: A map of human genome variation from population-scale sequencing. Nature, 2010; 467: 1061-1073

13) Wang G, Wang Z, Liang J, Cao L, Bai X, Ruan C: A phytosterolemia patient presenting exclusively with macrothrombocytopenia and stomatocytic hemolysis. Acta Haematol, 2011; 126: 95-98

14) Kishida D, Nakamura A, Yazaki M, Tsuchiya-Suzuki A, Matsuda M, Ikeda S: Genotype-phenotype correlation in Japanese patients with familial Mediterranean fever: differences in genotype and clinical features between Japanese and Mediterranean populations. Arthritis Res Ther, 2014; 16: 439

15) Escolà-Gil JC, Quesada H, Julve J, Martín-Campos JM, Cedó L, Blanco-Vaca F: Sitosterolemia: diagnosis, investigation, and management. Curr Atheroscler Rep, 2014; 16: 424

16) Neocleous V, Costi C, Kyriakou C, Kyriakides TC, Shammas C, Skordis N, Toumba M, Kyriakou S, Koliou M, Kousparou M, Onoufriou M, Hadjipanayis A, Iasonides M, Atamyan VN, Pierides A, Christophidou-Anastasiadou V, Tanteles GA, Phylactou LA: Familial Mediterranean fever associated with MEFV mutations in a large cohort of Cypriot patients. Ann Hum Genet, 2015; 79: 20-27

17) Tsubakio-Yamamoto K, Nishida M, Nakagawa-Toyama $Y$, Masuda D, Ohama T, Yamashita S: Current therapy for patients with sitosterolemia - effect of ezetimibe on plant sterol metabolism. J Atheroscler Thromb, 2010; 17: 891900

18) Niu DM, Chong KW, Hsu JH, Wu TJ, Yu HC, Huang CH, Lo MY, Kwok CF, Kratz LE, Ho LT: Clinical observations, molecular genetic analysis, and treatment of sitosterolemia in infants and children. J Inherit Metab Dis, 2010; 33: 437-443

19) $\mathrm{Hu}$ M, Yuen YP, Kwok JS, Griffith JF, Tomlinson B: Potential effects of NPC1L1 polymorphisms in protecting against clinical disease in a chinese family with sitosterolaemia. J Atheroscler Thromb, 2014; 21: 989-995 